\title{
Criterion for Dynamical Instability of Mass Transfer in Binary Evolution
}

\author{
Zhanwen Han \\ Yunnan Observatory, NAOC, CAS, Kunming 650011, P. R. China \\ Philipp Podsiadlowski \\ Astrophysics, NAPL, Oxford University, Keble Road, Oxford OX1 3RH, \\ $U K$
}

Christopher A. Tout

Institute of Astronomy, Madingley Road, Cambridge CB3 OHA, UK

\begin{abstract}
Using Eggleton's code, we performed a series of binary evolution calculations in order to investigate the criterion for dynamical instability of mass transfer in binaries. In these calculations, we took the donor's mass on the zero-age main sequence (ZAMS) from 0.8 to $1.9 M_{\odot}$. For each mass, we systematically varied the mass of the core at the beginning of mass transfer and the mass of the companion star. We assumed that mass transfer was completely non-conservative and that all the mass that was lost from the system carried with it the orbital angular momentum of the accreting component. We found that the critical mass ratio, above which mass transfer is dynamically unstable, is from 1.1 to 1.3 in these red-giant binary systems.
\end{abstract}

\section{Introduction}

Dynamical mass transfer in giant binaries may lead to the formation of many interesting objects and the criterion for dynamical instability of mass transfer therefore plays a very important role. In most binary evolution studies, the criterion is from polytropic models of stars. As is well known, if a fully convective star (modelled as a polytrope with a polytropic index $n=1.5$ ) loses mass its radius increases, while the Roche-lobe radius decreases if the mass donor is more massive than the accreting component. This means that the donor will overfill its Roche lobe by an ever increasing amount, leading to mass transfer on a dynamical timescale, the formation of a common envelope and a spiral-in phase. If mass transfer is conservative, the critical mass ratio is about $2 / 3$, i.e. mass transfer would be dynamically unstable if the donor has a mass larger than $2 / 3$ the mass of the companion star. However, this argument is not correct for a variety of reasons. First, it makes several severe simplifications. (1) Giant stars cannot be modelled as fully convective polytropes because they have massive degenerate cores. This increases the critical mass ratio for dynamical mass transfer 
substantially (Hjellming \& Webbink 1987). (2) the condition for dynamical instability also depends on the amount of mass and angular-momentum that is lost from the system (see e.g. Podsiadlowski, Joss, \& Hsu 1992; Han et al. 2001; Soberman, Phinney, \& van den Heuvel 1997). (3) Mass loss due to a stellar wind prior to the onset of mass transfer may significantly reduce the mass of the giant (and increase the fractional mass of the degenerate core). This mass loss could be significantly enhanced by tidal interaction with the companion (Eggleton \& Tout 1989). A second and perhaps even more fundamental problem with the simplistic application of such a criterion is that is does not take into account the detailed dynamics of the mass-transfer process, particularly during the turnon phase when a substantial amount of mass has already been lost before the dynamical instability sets in. Several recent full binary evolution calculations have shown that the simplistic criterion used in most binary population synthesis studies to date is not really appropriate; e.g. Tauris \& Savonije (1999) and Podsiadlowski, Rappaport, \& Pfahl (2002) have shown that, in the case of (sub-)giants transferring mass to a neutron star of $1.3 / 1.4 M_{\odot}$, mass transfer is dynamically stable for all giants up to a mass of about $2 M_{\odot}$ (see also Podsiadlowski et al. 1994 for an earlier example involving massive stars). On the observational side, it has long been clear that quite a few systems that should have experienced dynamical mass transfer and a CE phase appear to have been able to avoid it (see the discussion and references in Podsiadlowski et al. 1992).

In this paper, we investigate the criterion via detailed binary evolution calculations of red-giant binaries.

\section{Calculation}

Using the latest version of Eggleton's code, we performed a series of population I $(X=0.70, Y=0.28$ and $Z=0.02$ ) binary stellar evolution calculations for mass donors with different masses on the zero-age main sequence (ZAMS), ranging from 0.8 to $1.9 M_{\odot}$. For each mass we systematically varied the mass of the core at the beginning of mass transfer and the mass of the companion star. In these calculations we assumed that mass transfer was completely non-conservative and that all the mass that was lost from the system carried with it the orbital angular momentum of the accreting component (as appears to be most appropriate if the accretor is a white dwarf). In the standard set of calculations, we included a Reimers-type wind (Reimers 1975) with $\eta=1 / 4$ before the mass-transfer phase. In each calculation we checked whether mass transfer was dynamically stable. In cases where mass transfer is dynamically unstable there is no solution for the mass-transfer rate, $\dot{M}$, for which the radius of the secondary can be equal to the Roche-lobe radius (see Han, Tout, \& Eggleton 2000 for the treatment of the surface boundary condition). If mass transfer was stable, we continued mass transfer until the mass donor started to shrink below its Roche lobe, terminating the mass-transfer phase.

\section{Results}

The results of these calculations are summarized in Table 1 which shows, for each ZAMS mass, the core mass and the total mass of the donor at the beginning of 
Table 1. Critical masses for stable RLOF

\begin{tabular}{lllll}
\hline$M_{1}^{\text {ZAMS }}$ & $M_{c}$ & $M_{1}^{\mathrm{RLOF}}$ & $M_{2}^{\min }$ & $q_{\text {crit }}$ \\
$\left(M_{\odot}\right)$ & $\left(M_{\odot}\right)$ & $\left(M_{\odot}\right)$ & $\left(M_{\odot}\right)$ & \\
\hline 0.80 & 0.1992 & 0.7974 & 0.6243 & 1.2773 \\
0.80 & 0.2494 & 0.7945 & 0.6071 & 1.3087 \\
0.80 & 0.2996 & 0.7866 & 0.6071 & 1.2957 \\
0.80 & 0.3501 & 0.7683 & 0.6090 & 1.2616 \\
0.80 & 0.3994 & 0.7328 & 0.5888 & 1.2446 \\
0.80 & 0.4486 & 0.6696 & 0.5225 & 1.2815 \\
& & & & \\
1.00 & 0.1994 & 0.9975 & 0.8049 & 1.2393 \\
1.00 & 0.2498 & 0.9953 & 0.7898 & 1.2602 \\
1.00 & 0.2992 & 0.9894 & 0.7968 & 1.2417 \\
1.00 & 0.3493 & 0.9759 & 0.8229 & 1.1859 \\
1.00 & 0.3995 & 0.9496 & 0.8354 & 1.1367 \\
1.00 & 0.4482 & 0.9055 & 0.8185 & 1.1063 \\
& & & & \\
1.26 & 0.1994 & 1.2577 & 1.0339 & 1.2165 \\
1.26 & 0.2493 & 1.2560 & 1.0283 & 1.2214 \\
1.26 & 0.2993 & 1.2515 & 1.0452 & 1.1974 \\
1.26 & 0.3494 & 1.2414 & 1.0804 & 1.1490 \\
1.26 & 0.3985 & 1.2227 & 1.1149 & 1.0967 \\
1.26 & 0.4483 & 1.1905 & 1.1134 & 1.0692 \\
& & & & \\
1.60 & 0.2483 & 1.5968 & 1.3402 & 1.1915 \\
1.60 & 0.2991 & 1.5941 & 1.3366 & 1.1927 \\
1.60 & 0.3486 & 1.5873 & 1.3943 & 1.1384 \\
1.60 & 0.3981 & 1.5741 & 1.4494 & 1.0860 \\
1.60 & 0.4472 & 1.5522 & 1.4696 & 1.0562 \\
& & & & \\
1.90 & 0.2495 & 1.8969 & 1.5477 & 1.2256 \\
1.90 & 0.2963 & 1.8959 & 1.6066 & 1.1801 \\
1.90 & 0.3488 & 1.8920 & 1.6366 & 1.1561 \\
1.90 & 0.3982 & 1.8827 & 1.7322 & 1.0869 \\
\hline & & & &
\end{tabular}

Note - $M_{1}^{\text {ZAMS }}$ is the ZAMS mass of the primary; $M_{\mathrm{c}}$ is the core mass of the primary at the onset of RLOF; $M_{1}^{\mathrm{RLOF}}$ is the total mass of the primary at the onset of RLOF; $M_{2}^{\text {min }}$ is the minimum mass of the companion (WD/NS) for stable RLOF; $q_{\text {crit }}$ is the critical mass ratio. 

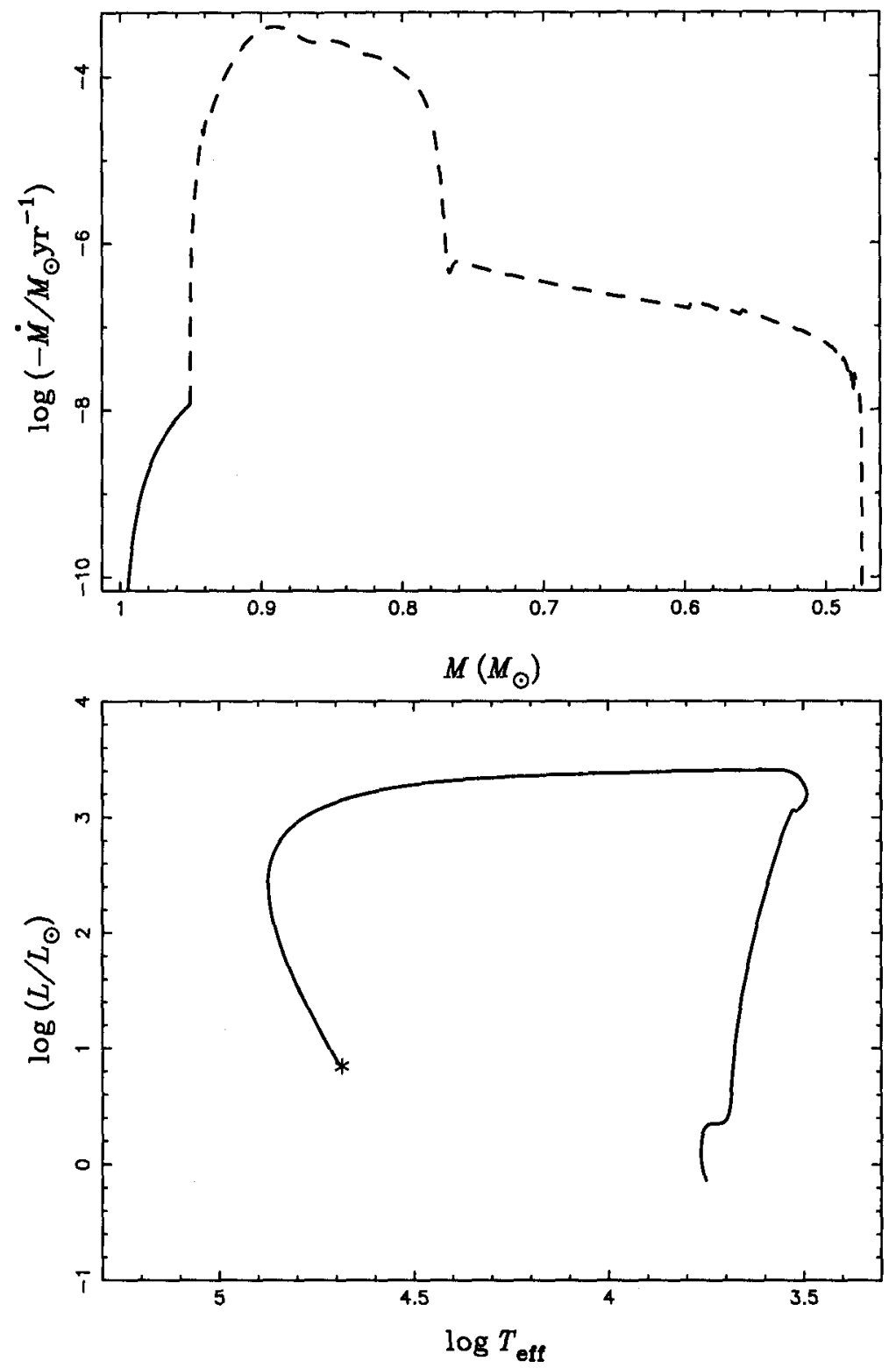

Figure 1. Evolution of mass-transfer rate (top panel) as a function of mass and evolutionary track in the H-R diagram (bottom panel) to demonstrate the case of stable RLOF for a binary with a giant donor with an initial mass of $1 M_{\odot}$ and a $0.84 M_{\odot}$ WD companion (Pop I, with overshooting, 1/4 Reimers' wind). The solid curve in the top panel shows the evolution before the onset of RLOF. This evolution is due to a stellar wind. No stellar wind was included during and after RLOF. 
mass transfer and the minimum mass of the secondary (and critical mass ratio) for which mass transfer is stable. These results demonstrate, as discussed above, that mass transfer is dynamically stable even if the mass donor is substantially more massive than the secondary. Note that, for each mass, the critical mass ratio tends to decrease for the initially more evolved systems because their evolutionary timescale is shorter and hence the mass-transfer rate higher than for the less-evolved ones. This has the consequence that the core mass grows less during the mass-transfer phase. One may also notice that some of the behaviour in Table 1 is non-monotonic. The non-monotonic behaviour of $q_{\mathrm{crit}}$ at the lowest $M_{\mathrm{c}}$ is caused by primaries with a core mass $M_{\mathrm{c}}$ near the base of the FGB, where the core is not very degenerate and the envelope is not yet fully convective. The non-monotonic behaviour for $0.8 M_{\odot}$ stars with the largest $M_{\mathrm{c}}$ is a consequence of the thin envelope mass.

Fig. 1 shows a representative binary calculation from one of the sequences for a star with a ZAMS mass of $1 M_{\odot}$. At the beginning of mass transfer the donor has a core mass of $0.3975 M_{\odot}$ and a total mass of $0.9508 M_{\odot}$ while the mass of the companion star is $0.84 M_{\odot}$. With these parameters, mass transfer starts at an orbital period of $348.4 \mathrm{~d}$. Initially, mass transfer occurs on a thermal timescale and reaches a maximum of $\sim 4 \times 10^{-4} M_{\odot} \mathrm{yr}^{-1}$. After the mass ratio has reversed and the star has regained thermal equilibrium, mass transfer settles to a rate of about $4 \times 10^{-7} M_{\odot} \mathrm{yr}^{-1}$ and gradually decreases as the secondary ascends the giant branch. Once the mass in the H-rich envelope drops below $0.021 M_{\odot}$, the donor shrinks inside its Roche lobe and mass transfer stops. As the remnant envelope collapses the star quickly moves across the H-R diagram and ultimately becomes a subdwarf B star (Han et al. 2002) of $0.4745 M_{\odot}$ in a wide binary with an orbital period of $948.9 \mathrm{~d}$.

\section{Discussion}

As we have shown in this paper, RLOF is more likely stable than previously believed. A stable RLOF leads to a wide binary consisting of a WD and the helium core of the giant. If the core is massive enough, helium can be ignited and the core becomes a subdwarf B star (Han et al. 2002). Subdwarf B stars produced this way will generally be in wide binaries with typical orbital periods of $400-1500 \mathrm{~d}$. This channel has been ignored in the past. However subdwarf B stars produced through this stable RLOF channel are consistent with the observations by Green, Liebert, \& Saffer (2001) who showed that some sdB stars appear to be members of long-period binaries.

In these full binary evolution calculations we assumed that mass transfer was completely non-conservative and that all the mass that was lost from the system carried with it the orbital angular momentum of the accreting component. We found that the critical mass ratio for stable RLOF $q_{\text {crit }} \approx 1.2$. For a polytropic model with $n=1.5$ this critical mass ratio would be $q_{\text {crit }}=1$.

In this paper, we assume completely non-conservative mass transfer to a WD companion for the stable RLOF. The assumption is appropriate for low mass-transfer rates, for which nova explosions are expected to expel the transferred matter, but possibly not for larger rates (Prialnik \& Kovetz 1995). For rates larger than about $10^{-7} M_{\odot} \mathrm{yr}^{-1}$ the white dwarf is able to accrete and 
steadily burn all the transferred matter (as in supersoft X-ray binaries), while for accretion rates approaching $10^{-6} M_{\odot} \mathrm{yr}^{-1}$ the accreted envelope begins to swell up (Nomoto, Nariai, \& Sugimoto 1979). In this case it is possible that most of the matter can indeed be expelled in an optically thick wind (Hachisu, Kato, \& Nomoto 1996) but such a model is not yet well established. There exists a further possibility that the white dwarf becomes a red giant as a result of accretion at $M>10^{-4} M_{\odot} \mathrm{yr}^{-1}$ (see Fig. 1). Even if this can be avoided, then during the second part of mass transfer in Fig. 1 the accretion rate is such that the WD mass can grow substantially. This would change the binary evolution.

The work presented here is part of our binary population synthesis project and a detailed study on the criterion is being carried out and will be published elsewhere.

Acknowledgments. This work partly supported by a Royal Society UKChina Joint Project Grant (PP and ZH), the Chinese National Science Foundation under Grant No. 19925312, 10073009 and NKBRSF No. 19990754 (ZH).

\section{References}

Eggleton, P. P., \& Tout, C. A. 1989, in Algols, ed. A. H. Batten (Dordrecht: Kluwer), 164

Green, E. M., Liebert, J., \& Saffer, R. A. 2001, in ASP Conf. Ser. Vol. 226, 12th European Workshop on White Dwarfs, ed. J. L. Provencal, H. L. Shipman, J. MacDonald, \& S. Goodchild (San Francisco: ASP), 192

Hachisu, I., Kato, M., \& Nomoto, K. 1996, ApJ, 470, L97

Han, Z., Eggleton P. P., Podsiadlowski, P., Tout, C. A., \& Webbink, R. F. 2001, in ASP Conf. Ser. Vol. 229, Evolution of Binary and Multiple Star Systems, ed. P. Podsiadlowski, S. Rappaport, A. R. King, F. D'Antona, \& L. Burderi (San Francisco: ASP), 205

Han, Z., Podsiadlowski, P., Maxted, P. F. L., Marsh, T. R., \& Ivanova, N. 2002, MNRAS, in press

Han, Z., Tout, C. A., \& Eggleton, P. P. 2000, MNRAS, 319, 215

Hjellming, M. S., \& Webbink, R. F. 1987, ApJ, 318, 794

Nomoto, K., Nariai, K., \& Sugimoto, D. 1979, PASJ, 31, 287

Podsiadlowski, P., Hsu, J. J. L., Joss, P. C., \& Ross, R. R. 1994, in The Late Stages of Stellar Evolution, ed. R. E. S. Clegg, I. R. Stevens, \& W. P. S. Meikle (Cambridge: CUP), 187

Podsiadlowski, P., Joss, P. C, \& Hsu, J. J. L. 1992, ApJ, 391, 246

Podsiadlowski, P., Rappaport, S., \& Pfahl, E. 2002, ApJ, 565, 1107

Prialnik, D., \& Kovetz, A. 1995, ApJ, 445, 789

Reimers, D. 1975, Mem. Roy. Soc. Sci. Liège, 8, 369

Soberman, G. E., Phinney, E. S., \& van den Heuvel, E. P. J. 1997, A\&A, 327, 620

Tauris, T. M., \& Savonije, G. J. 1999, A\&A, 350, 928

Webbink, R. F. 1988, in The Symbiotic Phenomenon, ed. J. Mikolajewska, M. Friedjung, S. J. Kenyon, \& R. Viotti (Dordrecht: Kluwer), 311 